\title{
Generation of cell-type-specific gene mutations by expressing the sgRNA of the CRISPR system from the RNA polymerase II promoters
}

\section{Dear Editor,}

Recently, the CRISPR/Cas9 system is emerging as a powerful tool for genome editing (Chang et al., 2013; Li et al., 2013; Niu et al., 2014; Shen et al., 2013; Wan et al., 2015; Wang et al., 2013) and genetic screening (Konermann et al., 2015), and holds great promise for biomedical applications in disease modeling and gene therapy by in vivo genome editing (Maddalo et al., 2014; Xue et al., 2014). However, the applications of CRISPR/Cas9 system still face some technical hurdles, one of which is to harness the gene editing in a precisely controlled manner. Of the two-component CRISPR/Cas9 system for genome editing, the Cas9 is a fixed genome-cutting component expressed from the RNA polymerase II (pol II) promoter that can drive tissue-specific gene expression; while the single-guide RNA (sgRNA) is a changeable genome-guiding component expressed from RNA polymerase III (pol III) promoter that usually drives the ubiquitous expression of "housekeeping" genes in all tissues. Therefore, to express the sgRNA in a tissue-specific manner can provide a convenient approach to tissue-specific gene mutations. Here, we reconstructed the sgRNA to enable its expression from the pol II promoters, and further achieved cell-type specific gene mutations via the modified CRISPR/Cas9 system by using celltype specific pol II promoters-driving sgRNA.

To generate pol II promoter-driving sgRNAs, we constructed a microRNA-shRNA-embedded sgRNA (miRshsgRNA) cassette that could express the small RNA from pol II promoter (Wang et al., 2007) into the 3 '-untranslational region (UTR) of the DsRed reporter gene (Figs. 1A and S1, and Supplementary Materials), as methods by cis-acting ribozymes (Gao \& Zhao, 2014; Nissim et al., 2014) and Cas6/ Csy4-based RNA processing (Nissim et al., 2014) have been reported. Notably, the nonsense shRNAs and a reported efficient sgRNA targeting the mouse p53 gene (sgp53) (Xue et al., 2014) was adopted in the construct for a proof-of-concept experiment. The mature sgRNA derived from the miRsh-sgRNA cassette will have additive 7 nucleotides in the $5^{\prime}$-end (Fig. 1A), so we chose a reported optimized sgRNA backbone, the sgRNA ${ }^{(F+E)}$, to improve the mutagenesis efficiency (Chen et al., 2013), given that the sgRNAs with mispairing and addition in the $5^{\prime}$-end are still functional (Cong et al., 2013).

To test whether functional sgRNA can be efficiently derived from the pol II promoter-driving miRsh-sgRNA cassette, we transfected mouse embryonic fibroblasts (MEFs) and mouse embryonic stem cells (mESCs) with constitutive EF1a promoter-driving miRsh-sgp53 expression vector and Cas9-P2A-EGFP expression vector. GFP and DsRed double positive cells were sorted by fluorescence-activated cell sorting (FACS) two days after transfection for further analysis (Fig. 1B). The T7EN1 cleavage assay of these cells showed that constitutive EF1a promoter-driving miRsh-sgp53 can guide Cas9 for producing double strand breaks (DSBs) in p53 gene in both MEFs and mESCs (Fig. 1C). To confirm the T7EN1 cleavage results, Sanger sequencing was performed and the result showed that the efficiency of miRsh-sgp53 in MEFs (Fig. 1D) and mESCs (Fig. 1E) was about 50\% (6/12) and $57.1 \%(8 / 14)$. These results indicated that the functional sgRNA could be expressed from the pol II promoter-driving miRsh-sgRNA construct for successful gene mutations.

Further, to examine whether the miRsh-sgRNA cassette can produce gene mutation in a cell type-specific manner, we constructed an expression vector using the embryonic stem cell-specific mouse Oct4 gene promoter (mOct4P) to express the miRsh-sgp53 cassette. Two days after transfection of MEFs and mESCs with this vector and the EF1a promoter-driving Cas9-P2A-EGFP vector, GFP and DsRed double-positive mESCs were observed, but only GFP positive MEFs were observed, indicating the cell-type-specific expression of the sgRNA (Fig. 1F). We sorted GFP and DsRed double positive mESCs and GFP positive MEFs by FACS for further analysis. T7EN1 cleavage assay and Sanger sequencing were performed, and $p 53$ gene mutation was only detected in the mESCs but not in the MEFs (Fig. 1G). The result of Sanger sequencing showed that the 


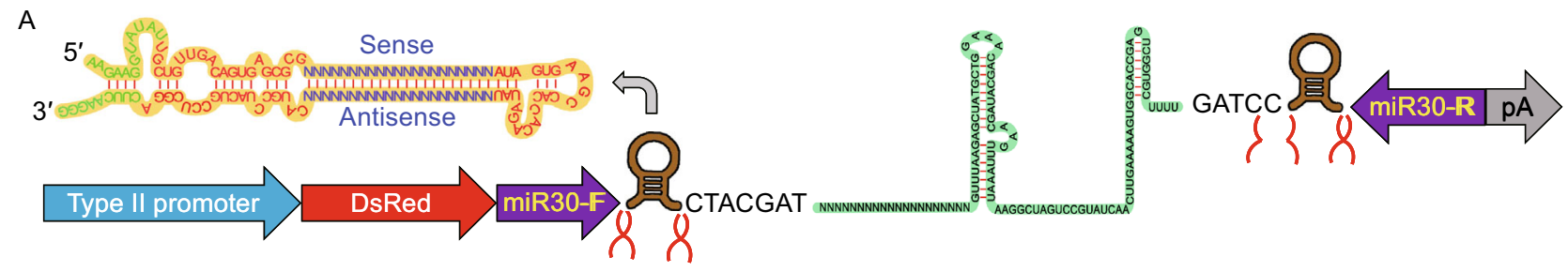

B
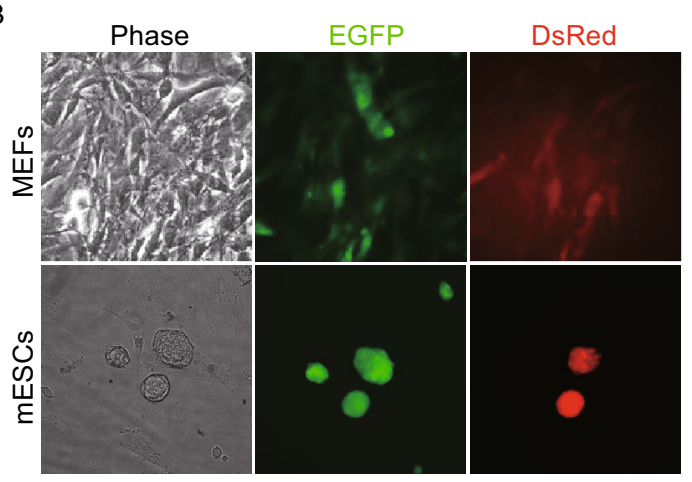

$\mathrm{F}$

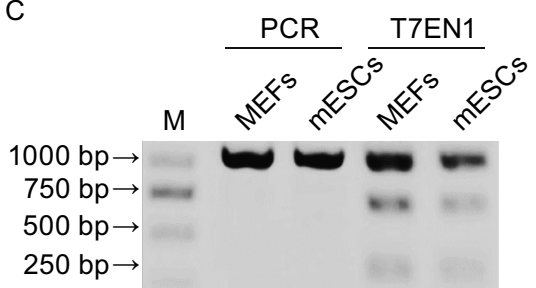

G

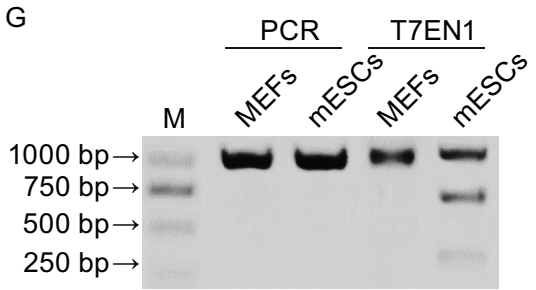

D ACAGTCGGATATCAGCCTCGAGCTCCCTCTGAGCCAGGAGACAT ACAGTCGGATATCAGCCTCGAGCTCCCT TTGAGCCAGGAGACAT ACAGTCGGATATCAGCCTCGAGCTCCCT t $t$ TGAGCCAGGAGACAT ACAGTCGGATATCAGCCatcagtagcaTCCCTCTGAGCCAGGAGACAT ACAGTCGGATATCAGCCTCGAGCTCCCT t t t TGAGCCAGGAGACAT ACAGTCGGATATCAGCCTCGAGCT---T-TGAGCCAGGAGACATT

E

ACAGTCGGATATCAGCCTCGAGCTCCCTCTGAGCCAGGAGACAT ACAGTCGGATATCAGCCTCGAGCTCCCTCC TGAGCCAGGAGACAT ACAGTCGGATATCAGCCTCGAGCTCCC CCCCAGGAGACAT ACAGTCGGATATCAGCCTCGAGCTCCCe $t$ TTGAGCCAGGAGACAT ACAGTCGGATATCAGCCTacagatgaCTGAGCCAGGAGACAT ACAGTCGGATATCAGCCTCGAGCT------GAGCCAGGAGACAT ACAGTCGGATATCAGCCTCGAG

$\mathrm{H}$

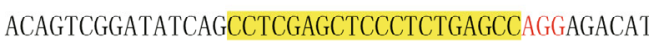
ACAGTCGGATATCAGCCTCGAGCTCCCTC CaTGAGCCAGGAGACAT ACAGTCGGATATCAGCCTCGAGCTCCCTCTGGGCCAGGAGACAT ACAGTCGGATATCAGCCTCGAGC-CCC-C--- CCAGGAGACAT ACAGTCGGATATCAG------_------_------ GACAT A--------------------------GAGCCAGGAGACAT
DsRed

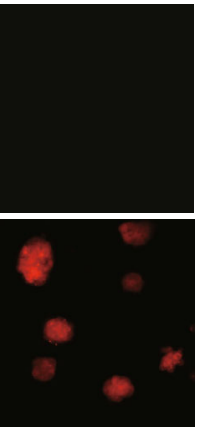

Wide-type

$\mathrm{C} \rightarrow \mathrm{T} \quad[\times 2]$

$(-1,+12)$

$(-6,+10)$

$(-1,+3)$

(-4) $\quad 6 / 12$

Wide-type

(+1) [×3]

$(-6,+3)$

$(-2,+3)$

$(-10,+8)$

$(-6)$

(-21) $8 / 14$

Wide-type

(+2) [×4]

$A \rightarrow G$

$(-6)[\times 2]$

$(-24)$

(-29) $9 / 17$
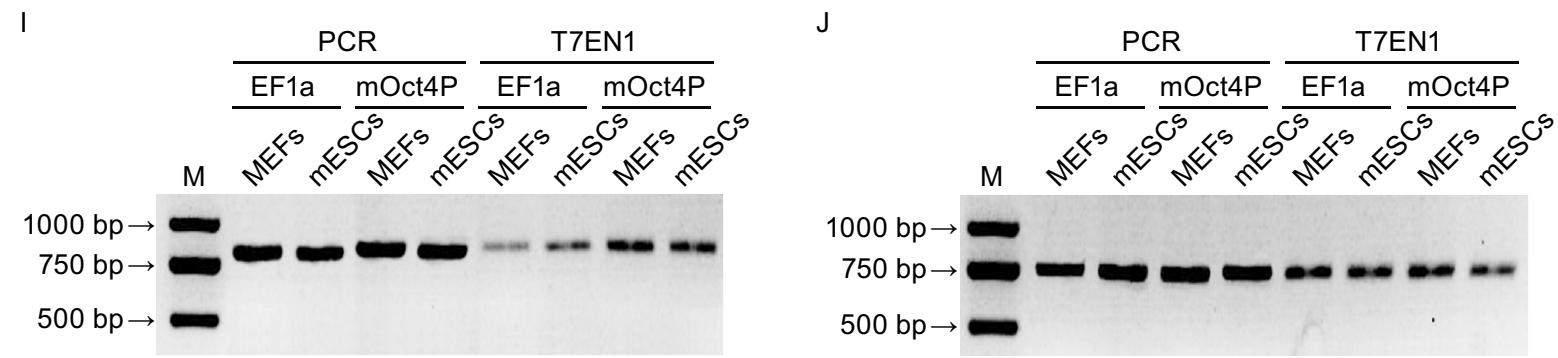
Figure 1. Pol II promoter-driving miRsh-sgp53 functions in MEFs and mESCs. (A) Structure of pol II promoter-driving miRsh-sgRNA cassette. The cut sites of Drasha in this primarymicroRNA are marked, and the resulted sgRNA would have 7 additive nucleotides (CTACGAT) at the $5^{\prime}$-end. (B) MEFs and mESCs transfected with Cas9-EGFP and constitutive EF1a promoter-driving DsRed-miRsh-sgp53 vectors are both GFP and DsRed positive at $48 \mathrm{~h}$ after transfection. (C) T7EN1 cleavage assay showed that constitutive EF1a promoter-driving miRsh-sgp53 can guide Cas9 for producing DSBs in both MEFs and mESCs. The PCR band is $972 \mathrm{bp}$ and the theoretical cut bands are $286 \mathrm{bp}$ and $686 \mathrm{bp}$. ( $\mathrm{D}$ and E) Representative Sanger sequencing results of the PCR amplicons from MEFs (D) or mESCs $(E)$ transfected with EF1a-driving miRsh-sgp53 cassette. AGG (red) is the protospacer-adjacent motif (PAM) sequence. Mutations were described in brackets. (F) MEFs transfected with Cas9-EGFP and stem cell-specific mOct4P promoter-driving DsRed-miRsh-sgp53 vectors are only GFP positive while mESCs are both GFP and DsRed positive at $48 \mathrm{~h}$ after transfection. (G) T7EN1 cleavage assay showed that stem-cell-specific mOct4P promoter-driving miRsh-sgp53 can guide Cas9 for producing DSBs only in mESCs but not in MEFs. The PCR band is $972 \mathrm{bp}$ and the theoretical cut bands are $286 \mathrm{bp}$ and $686 \mathrm{bp}$. $(\mathrm{H})$ Representative Sanger sequencing results of the PCR amplicons from mESCs transfected with mOct4P-driving miRsh-sgp53 cassette. AGG (red) is the protospacer-adjacent motif (PAM) sequence. Mutations were described in brackets. (I) Off targeting analysis of p53-off-27 site in MEFs and mESCs transfected with EF1a-driving miRshsgp53 and Cas9 or mOct4P-driving miRsh-sgp53 and Cas9 by T7EN1 assay. The PCR band is $800 \mathrm{bp}$ and the theoretical cut bands are $315 \mathrm{bp}$ and $485 \mathrm{bp}$. (J) Off targeting analysis of p53off-28 site. The PCR band is $753 \mathrm{bp}$ and the theoretical cut bands are $281 \mathrm{bp}$ and $472 \mathrm{bp}$.

p53 mutation efficiency in mESCs with ESC-specific mOct4P-driving miRsh-sgp53 $(52.9 \%, 9 / 17)$ was similar to that with constitutive EF1a promoter-driving sgp53 (Fig. $1 \mathrm{H}$ ). These results indicated that the cell type-specific gene editing can be achieved by cell type-specific promoter-driving expression of miRsh-sgRNA.

Previous studies suggested that CRISPR/Cas9 system would probably induce off-target mutations because the binding to genome could tolerate sequence mismatches distal from the PAM at the $5^{\prime}$ end of sgRNAs. It has been demonstrated in bacteria and cultured human cells that the DNA cleavage specificity of CRISPR/Cas9 system is determined by the PAM sequence NGG and the 8-12 base "seed sequence" at the $3^{\prime}$ end of the sgRNA (Cong et al., 2013; Jinek et al., 2013; Wang et al., 2013). We searched for potential off target sites based on this rule, and found 41

Jiaqiang Wang and Xin Li have contributed equally to this work.

Electronic supplementary material The online version of this article (doi:10.1007/s13238-015-0169-x) contains supplementary material, which is available to authorized users. potential off targets of the sgp53 (named p53-off-1 to p53-off41) existing in mouse genome (Table S2). The T7EN1 assay and Sanger sequencing revealed that no potential off target site we tested (Figs. 1I, 1J and S2) was mutated by miRshsgp53 in all the examined cells.

In conclusion, we designed a new construct for efficient and visible expression of sgRNAs from the pol II promoters, which therefore can produce cell-type specific mutations. This reconstructed pol II promoter-driving miRsh-sgRNA backbone will make the CRISPR/Cas9 system-mediated genome editing be more controllable and safer for future applications such as in in vivo gene therapy.

\section{FOOTNOTES}

The authors thank all members of Laboratory of Embryo Biotechnology in College of life science in Northeast Agricultural University and State Key Laboratory of Reproductive Biology in Institute of Zoology in Chinese Academy of Sciences; this study was supported by the grant from the International S\&T Cooperation Programme 2013DFG30680, the grant from the "Strategic Priority Research Program" of the Chinese Academy of Sciences XDA01010407, and the grant from the National Basic Research Program (973 Program) (Nos. 2011CBA01006 and 2011CB944202). We thank Ting Li, Qing Meng and Hua Qin for their help with fluorescence-activated cell sorting.

Jiaqiang Wang, Xin Li, Yanhua Zhao, Qi Zhou and Zhonghua Liu declare that they have no conflict of interest.

This article does not contain any studies with human or animal subjects performed by the any of the authors.

Jiaqiang Wang ${ }^{1}$, Xin $\mathrm{Li}^{1,2}$, Yanhua Zhao ${ }^{1}$, Jingyu $\mathrm{Li}^{1}$, Qi Zhou ${ }^{1,2 \bowtie}$, Zhonghua Liu ${ }^{1 凶}$

${ }^{1}$ College of Life Sciences, Northeast Agricultural University, Harbin 150030, China

2 State Key Laboratory of Reproductive Biology, Institute of Zoology, Chinese Academy of Sciences, Beijing 100101, China

\section{OPEN ACCESS}

This article is distributed under the terms of the Creative Commons Attribution 4.0 International License (http://creativecommons.org/ licenses/by/4.0/), which permits unrestricted use, distribution, and reproduction in any medium, provided you give appropriate credit to the original author(s) and the source, provide a link to the Creative Commons license, and indicate if changes were made.

\section{REFERENCES}

Chang N, Sun C, Gao L, Zhu D, Xu X, Zhu X, Xiong JW, Xi JJ (2013) Genome editing with RNA-guided Cas9 nuclease in Zebrafish embryos. Cell Res 23:465-472

Chen B, Gilbert LA, Cimini BA, Schnitzbauer J, Zhang W, Li GW, Park J, Blackburn EH, Weissman JS, Qi LS, Huang B (2013) 
Dynamic imaging of genomic loci in living human cells by an optimized CRISPR/Cas system. Cell 155:1479-1491

Cong L, Ran FA, Cox D, Lin S, Barretto R, Habib N, Hsu PD, Wu X, Jiang W, Marraffini LA, Zhang $F$ (2013) Multiplex genome engineering using CRISPR/Cas systems. Science 339:819-823

Gao Y, Zhao Y (2014) Self-processing of ribozyme-flanked RNAs into guide RNAs in vitro and in vivo for CRISPR-mediated genome editing. J Integr Plant Biol 56:343-349

Jinek M, East A, Cheng A, Lin S, Ma E, Doudna J (2013) RNAprogrammed genome editing in human cells. eLife 2:e00471

Konermann S, Brigham MD, Trevino AE, Joung J, Abudayyeh OO, Barcena C, Hsu PD, Habib N, Gootenberg JS, Nishimasu H, Nureki O, Zhang F (2015) Genome-scale transcriptional activation by an engineered CRISPR-Cas9 complex. Nature 517:583588

Li W, Teng F, Li T, Zhou Q (2013) Simultaneous generation and germline transmission of multiple gene mutations in rat using CRISPR-Cas systems. Nat Biotechnol 31:684-686

Maddalo D, Manchado E, Concepcion CP, Bonetti C, Vidigal JA, Han YC, Ogrodowski P, Crippa A, Rekhtman N, de Stanchina E, Lowe SW, Ventura A (2014) In vivo engineering of oncogenic chromosomal rearrangements with the CRISPR/Cas9 system. Nature 516:423-427

Nissim L, Perli SD, Fridkin A, Perez-Pinera P, Lu TK (2014) Multiplexed and programmable regulation of gene networks with an integrated RNA and CRISPR/Cas toolkit in human cells. Mol Cell 54:698-710
Niu Y, Shen B, Cui Y, Chen Y, Wang J, Wang L, Kang Y, Zhao X, Si W, Li W, Xiang AP, Zhou J, Guo X, Bi Y, Si C, Hu B, Dong G, Wang H, Zhou Z, Li T, Tan T, Pu X, Wang F, Ji S, Zhou Q, Huang X, Ji W, Sha J (2014) Generation of gene-modified cynomolgus monkey via Cas9/RNA-mediated gene targeting in one-cell embryos. Cell 156:836-843

Shen B, Zhang J, Wu H, Wang J, Ma K, Li Z, Zhang X, Zhang P, Huang $X$ (2013) Generation of gene-modified mice via Cas9/ RNA-mediated gene targeting. Cell Res 23:720-723

Wan H, Feng C, Teng F, Yang S, Hu B, Niu Y, Xiang AP, Fang W, Ji W, Li W, Zhao X, Zhou Q (2015) One-step generation of p53 gene biallelic mutant cynomolgus monkey via the CRISPR/Cas system. Cell Res 25:258-261

Wang J, Theunissen TW, Orkin SH (2007) Site-directed, virus-free, and inducible RNAi in embryonic stem cells. Proc Natl Acad Sci USA 104:20850-20855

Wang H, Yang H, Shivalila CS, Dawlaty MM, Cheng AW, Zhang F, Jaenisch R (2013) One-step generation of mice carrying mutations in multiple genes by CRISPR/Cas-mediated genome engineering. Cell 15:910-918

Xue W, Chen S, Yin H, Tammela T, Papagiannakopoulos T, Joshi NS, Cai W, Yang G, Bronson R, Crowley DG, Zhang F, Anderson DG, Sharp PA, Jacks T (2014) CRISPR-mediated direct mutation of cancer genes in the mouse liver. Nature 514:380-384 